\title{
Pt/Au based Sensor with a PMMA -Film for detecting CO in a Hydrogen Atmosphere
}

\author{
S. Simon $^{1}$, R. Marjunus ${ }^{1,4}$, T. Stimpel - Lindner ${ }^{1}$, Ch. Wilbertz ${ }^{2}$, I. Schmidt ${ }^{3}$, J. Wartmann ${ }^{3}$, W. Hansch ${ }^{1}$ \\ ${ }^{1}$ Universität der Bundeswehr München - Institute of Physics, Germany \\ Stefan.Simon@unibw.de \\ ${ }^{2}$ Micronas GmbH, Freiburg - Germany \\ ${ }^{3}$ Zentrum für Brennstoffzellentechnik, Duisburg - Germany \\ ${ }^{4}$ University of Lampung - Department of Physics, Indonesia
}

\begin{abstract}
:
Even small traces of carbon monoxide in the fuel gas lead to a degradation of performance in low temperature polymer electrolyte membrane (PEM) fuel cell systems. Therefore a sensor which is capable of detecting $\mathrm{CO}$ in a ppm range in a hydrogen rich atmosphere is a great advantage. In this talk a new gas sensitive layer to be used as suspended gate in an existing CCFET based sensor system is presented. The new gate material is based on a platinum/gold alloy and covered with a thin polymethylmethacrylat (PMMA) layer. A simple experiment was designed to qualify the sensitive layer measuring the work function change with a Kelvin probe system. Here measurements will be presented in an atmosphere of $95 \%$ argon, $4 \%$ hydrogen and $1 \%$ carbon dioxide. The carbon monoxide concentration was up to 50ppm.
\end{abstract}

Key words: Carbon monoxide, fuel cell, sensor, hydrogen, platinum/gold alloy.

\section{Introduction}

Hydrogen for fuel cells can be produced by a reformation process of hydrocarbons and water. At high temperatures up to $900{ }^{\circ} \mathrm{C}$ and pressures around 25 bar, water and hydrocarbons react to hydrogen. As a byproduct of this process carbon monoxide (up to $1 \%$ ) is derived. CO is highly poisonous for the platinum catalyst layer of the fuel cell membrane, what leads to a degradation of the fuel cell performance [1]. A reduction of $\mathrm{CO}$ concentration to a few ppm in the fuel gas is essential. This can be reached by selective oxidation and purification processes [2]. But for these applicable methods, it is important that the amount of $\mathrm{CO}$ is known.

Nowadays, many different gas sensing methods exist [3]. A well suited way to detect gases is using the Floating Gate Field Effect Transistor (FGFET) [4]. Its sensing properties depend on the gas - induced work function change (or Contact Potential Difference - CPD) of the sensitive layer that is mounted on top of the transducer device. A related CCFET based Sensor system with convertible gas sensitive layers on a suspended gate, mySens ${ }^{\circledR}$, has been developed by Micronas $\mathrm{GmbH}$ in Freiburg. The goal of the presented work is to develop a gas sensitive layer which can detect $\mathrm{CO}$ in hydrogen-rich atmosphere, and which can be used with the mySens ${ }^{\circledR}$ technology as a $\mathrm{CO}$ sensor for fuel cell systems.

$\mathrm{CO}$ and $\mathrm{H}_{2}$ are reducing gases. So, the reaction of the two gases on surfaces is quite similar in principle. Up today, some studies of $\mathrm{CO}$ detection in $\mathrm{H}_{2}$ have been done $[5,6,7]$. In the present article a sensitive layer based on gold alloyed platinum will be presented, able to detect carbon monoxide in a hydrogen rich atmosphere. On top of its surface, additionally a thin polymethylmethacrylat (PMMA) film was placed as a diffusion barrier [8]. The gas induced work function change was measured with a kelvin probe system. The measurement gas conditions were $4 \% \mathrm{H}_{2}, 1 \% \mathrm{CO}_{2}$ and up to 50 ppm CO concentration. Argon was used as an inert carrier gas.

\section{Sample Preparation}

In this work, highly doped $<100>$ silicon wafers with specific resistances between $0.01 \Omega \mathrm{cm}$ and $0.5 \Omega \mathrm{cm}$ were used as carrier materials. On top of these wafers, $100 \mathrm{~nm}$ titanium was sputtered as adhesive layer with a LeyboldHeraeus Z550 sputtering device. In the next step, a $100 \mathrm{~nm}$ thick platinum-gold layer was sputtered with a Polaron SC500 SputterCoater. 
For this, a Pt/Au mixed sputter target was used, built up on a round metal holder with two semicircular foils of platinum and gold with a purity of $99,9 \%$ and on the other side a semicircular gold foil with a purity of $99,9 \%$ each. Both foils were delivered from the AlfaAesar Company. Due to the split configuration of the sputter target, we received a gradient of the $\mathrm{Pt} / \mathrm{Au}$ ratio across the sputtered wafer. After the sputtering process, the wafers were divided in small square pieces with a side length of about one $\mathrm{cm}$. The compounding of these different samples was investigated with Energy Dispersive X-ray Spectroscopy (EDX) by an INCA 2 X-act EDXsystem from Oxford Instruments built in a JEOL JSM-6700F Scanning Electron Microscope (SEM). In the next step, the PMMA (photo resistive AR-PC504, Allresist) was deposited on these small samples by spin coating. This PMMA was diluted down to $33 \%$ with the diluent AR600-01 from the same manufacturer. After the depositing of PMMA, the sample was spun $5 \mathrm{sec}$ at $500 \mathrm{rpm}$ and then $25 \mathrm{sec}$ at 8000 rpm to get a smooth homogeneous about 100 $\mathrm{nm}$ thick layer. After baking out the samples for $10 \mathrm{~min}$ at $180{ }^{\circ} \mathrm{C}$ on a conventional hotplate, the thickness was investigated with a Micropack NanoCalc2000 spectrometer. To get thinner PMMA layers, the surface was etched in oxygen plasma with a Technics Planar ETCH II plasma etching system. In a last step, the thickness of the PMMA was checked again.

\section{Experimental}

The samples were characterized in the gas measurement station, schematically shown in Fig. 1. It is a closed chamber with a volume of about $12 \mathrm{~cm}^{3}$. Inside the chamber the samples were glued with conductive silver on a grounded sample holder opposite a kelvin control probe. The sample holder could be heated up to $150{ }^{\circ} \mathrm{C}$. The temperature was measured with a Pt100 temperature sensor. The gas flow was controlled by a WIGHA2000 control unit and three MKS mass flow controllers (MFC). For our measurement a stable gas flow of $100 \mathrm{sccm}$ gas was established. Two different test gases supplied by the Linde AG were used. One gas used as background atmosphere consisted of $4 \% \mathrm{H}_{2}$, $1 \% \mathrm{CO}_{2}$ and $95 \% \mathrm{Ar}$. The other one contained $4 \% \mathrm{H}_{2}, 1 \% \quad \mathrm{CO}_{2}, 95 \% \mathrm{Ar}$ and additionally 200 ppm carbon monoxide. With these two gases it was possible to vary the carbon monoxide concentration from 0 to 200 ppm with a constant hydrogen background. An additional bubbler line enabled humidity variations in the test gas up to $50 \%$. The CPD was measured by a Kelvin Control 07 Unit from the Besocke Delta
Phi GmbH connected to a Keithley2000 multimeter which monitored the CPD. The whole measurement station was controlled by an in house developed MatLab program on a conventional laptop.

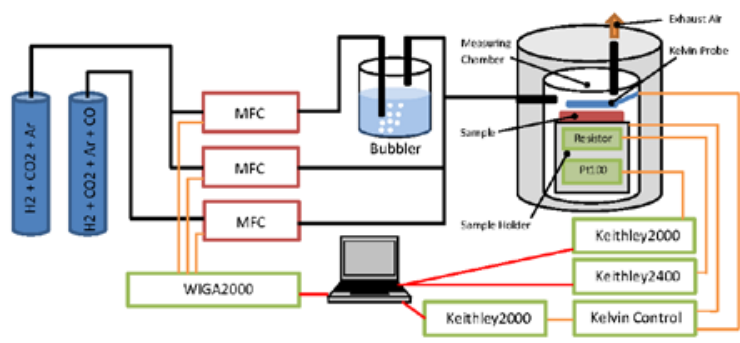

Fig. 1: Schematic diagram of the used measurement station.

\section{Results}

The first measurements were done with pure platinum layers. The results are plotted in Fig. 2. The upper part of the graphic shows the CPD of the platinum layers. The lower part describes the measurement conditions (temperature, relative humidity and $\mathrm{CO}$ concentration) during the measurement. During the CO-peaks a CPD change was measured but the signal behaviors are not constant over all measurement conditions. Especially at low temperatures $\sim 25^{\circ} \mathrm{C}$ the CPD is very small. Furthermore you can see a large back drift in the middle temperature range with 50\% humidity.

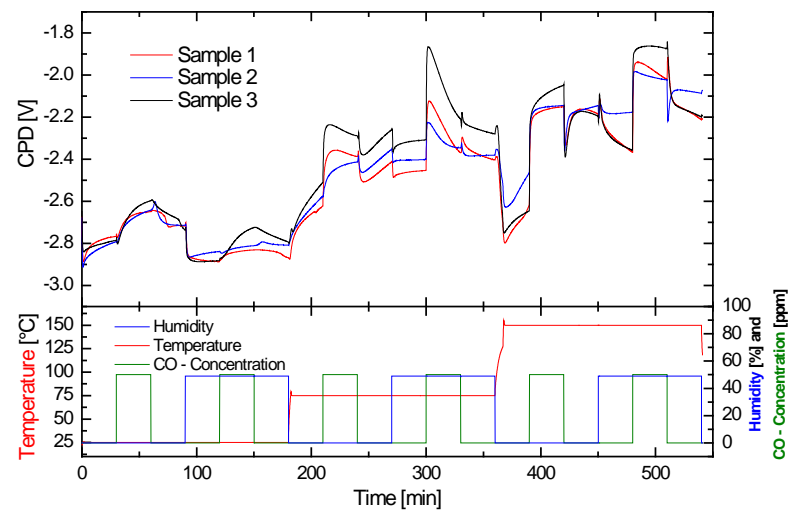

Fig. 2: Kelvin probe measurements of a pure platinum layer at different temperatures with an without humidity in a 4\% hydrogen atmosphere with 95\% argon, 1\% carbon dioxide and a maximum concentration of $50 \mathrm{ppm}$ carbon monoxide.

Now we tried to improve the sensor signals. Therefore we produced an alloy of platinum and gold. In Fig. 3 the CPD of platinum gold alloys with different $\mathrm{Pt} / \mathrm{Au}$ concentrations is shown. The signal peaks of the $\mathrm{Pt} / \mathrm{Au}$ alloys are noticeable over the whole temperature range without pronounced humidity influence. 
Regarding the $\mathrm{Pt} / \mathrm{Au}$ ratio a high concentration of platinum (about 90\% - black line) gives better signals of about $200 \mathrm{mV}$ in comparison to samples with a high gold amount (blue line). The latter do not show a constant reaction.

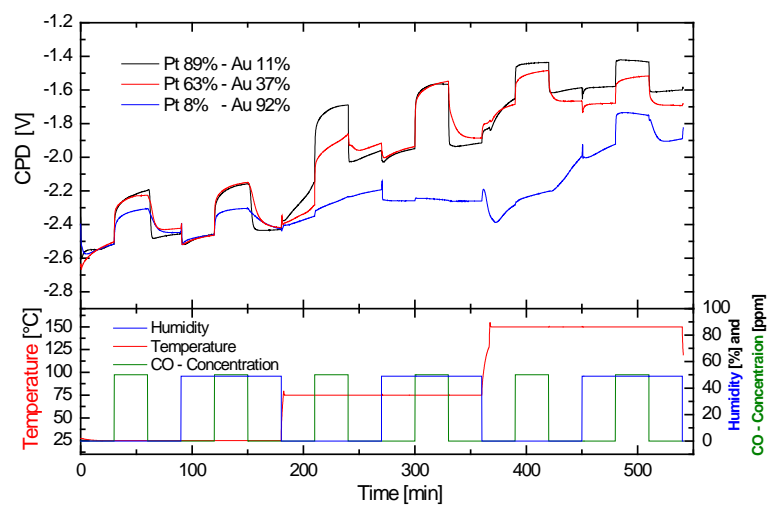

Fig.3: Kelvin probe measurements of Pt/Au alloys under the same measurements conditions as above.

The next improvement step was, to increase the signal discrimination of different $\mathrm{CO}$ concentrations. On pure Pt/Au alloys, the peak heights of the CPD for certain CO amounts cannot be distinguished clearly. We get only a few $\mathrm{mV}$ differences between $10 \mathrm{ppm}$ and 100 ppm CO as you can see in Fig.4.

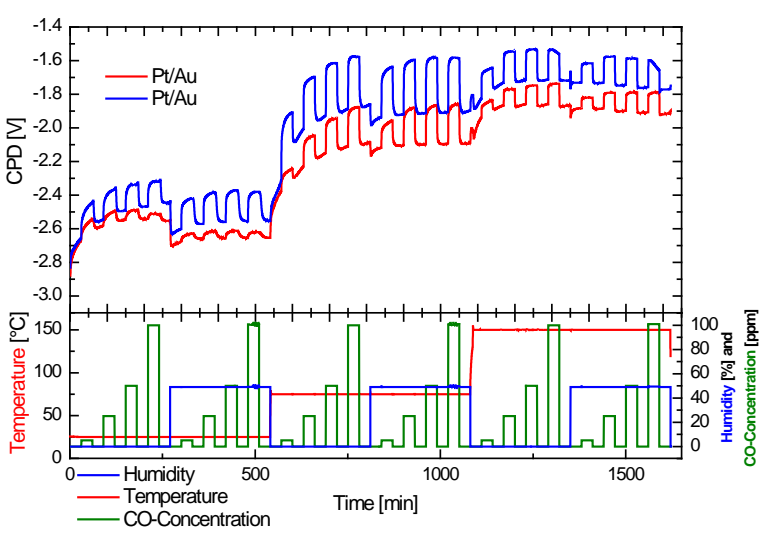

Fig.4: Kelvin probe measurements of Pt/Au layers at different CO-concentration up to $100 \mathrm{ppm} \mathrm{CO}$. No clear difference between the CPD changing is recognizable.

Inspired by previous sensor developments [8], the Pt/Au alloy was covered with a PMMAlayer. The results are printed in Fig. 5. Both samples have approximately the same $\mathrm{Pt} / \mathrm{Au}$ ratio 9:1. The thicknesses of the PMMA-layers were $11 \mathrm{~nm}$ and $16 \mathrm{~nm}$. For thicker PMMAlayers of about $30 \mathrm{~nm}$ the $\mathrm{CO}$ signal disappeared completely. This system was able to detect different $\mathrm{CO}$ concentrations. Especially at high operation temperatures of $150{ }^{\circ} \mathrm{C}$ a clear difference in the CPD signals of $\mathrm{CO}$ amounts of $10 \mathrm{ppm}, 25 \mathrm{ppm}$ or $50 \mathrm{ppm}$ was measurable.

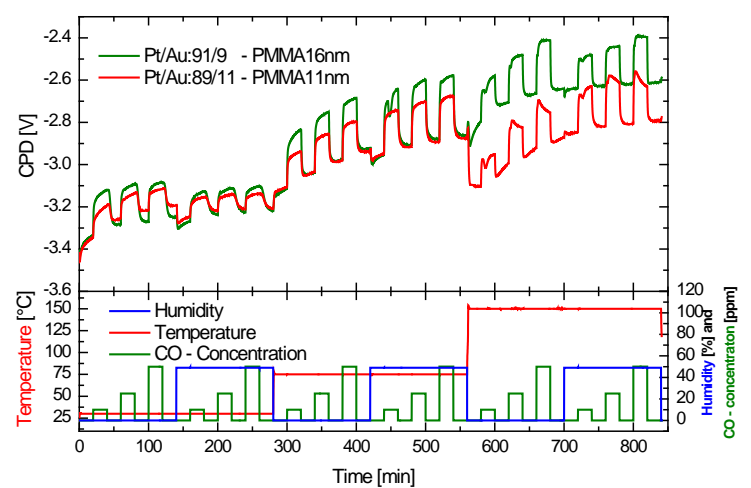

Fig.5: Kelvin probe measurements of Pt/Au alloys with an additional PMMA layer on top. The CO concentration was varied form $10 \mathrm{ppm}$ to $50 \mathrm{ppm}$.

\section{Conclusion}

With these measurements, we have shown that it is not only possible to detect $\mathrm{CO}$ in hydrogen containing atmosphere by the CPD measurement of a platinum gold alloy with a high fraction of platinum. Moreover we can distinguish different concentrations of this gas, when an additional PMMA-layer of about $15 \mathrm{~nm}$ thickness covers the Pt/Au surface. In the next step, the sensitive layer will be tested in higher hydrogen concentrations up to $80 \%$ and the cross sensitivities to other gases like methane, carbon dioxide and oxygen will be checked.

\section{ZIM - Project}

The development of this sensor layer is based on a cooperation of Micronas $\mathrm{GmbH}$, enyMotion $\mathrm{GmbH}$, the fuel cell research center ZBT GmbH and the Universität der Bundeswehr München Germany supported by the Federal Ministry of Economics and Technology on the basis of a decision by the German Bundestag.

\section{References}

[1] Xuan Cheng, Zheng Shi, Nancy Glass, Lu Zhang, Jiujun Zhang, Datong Song, Zhong-Sheng Liu, Haijiang Wang, and Jun Shen. A review of pem hydrogen fuel cell contamination: Impacts, mechanisms, and mitigation. Journal of Power Sources, 165(2):739 - 756, 2007

[2] H. Beyer, B. Schönbrod, C. Siegel, M. Steffen, A. Heinzel, Two-dimensional Modelling of a Nonisothermal PROX Reactor with Water Cooling for Fuel Cell Applications, European COMSOL Conference 2009, 14-16. Oct. 2009, Milan, Italy

[3] S. Capone, A. Forelo, L. Francioso, R. Rella, P. Siciliano, J. Spadavecchia, D. S. Presicce, and A. AmTaurino. Solid state gas sensors: State of the art and future activities. Journal of Optoelectronics and Advanced Materials, 5(5):1335 - 1348, 2003.

[4] M. Burgmair, H.-P. Frerichs, M. Zimmer, M. Lehmann, and I. Eisele, Field effect transducers for work function gas measurements: device 
improvement and comparison of performance. Sensors and Actuators B: Chemical, 95(1-3):183 - 188, 2003.

[5] Christopher T Holt, A.-M Azad, S.L Swartz, R.R Rao, and Prabir K. Dutta. Carbon monoxide sensor for pem fuel cell systems. Sensors and Actuators B: Chemical, 87(3):414 - 420, 2002.

[6] Atsuko Hashimoto, Takashi Hibino, and Mitsuru Sano. Solid oxide fuel cells that enable the detection of $\mathrm{CO}$ in reformed gases. Sensors and Actuators B: Chemical, 86(1):12 - 19, 2002.

[7] Hiroyuki Yamaura, Yuji Iwasaki, Shinsuke Hirao, and Hidenori Yahiro. Cuo/sno2-in2o3 sensor for monitoring $\mathrm{CO}$ concentration in a reducing atmosphere. Sensors and Actuators B: Chemical, 153(2):465 - 467, 2011.

[8] C. Senft, P. Iskra, I. Eisele, C. Wilbertz, and H.P. Frerichs. Temperature controlled phase transition as a detection principle for gas fets (tpt-fet). Sensors, 2008 IEEE, pages $1316-1319$, oct. 2000 